TRANSACTIONS OF THE

AMERICAN MATHEMATICAL SOCIETY

Volume 363, Number 1, January 2011, Pages 439-455

S 0002-9947(2010)05181-5

Article electronically published on August 31, 2010

\title{
KAC-WAKIMOTO CHARACTERS AND UNIVERSAL MOCK THETA FUNCTIONS
}

\author{
AMANDA FOLSOM
}

\begin{abstract}
In recent work, Bringmann and Ono answer a question of Kac and show that character formulas for $s \ell(r+1,1)^{\wedge}$ modules due to Kac and Wakimoto are "holomorphic parts" of nonholomorphic modular functions. Here, we confirm a speculation of Ono that these characters are, up to a simple $q$-series, the universal mock theta functions $g_{2}(\omega, q)$ and $g_{3}(\omega, q)$ of Gordon and McIntosh. Using recent work of Bringmann-Ono, Kang, Zwegers, and Gordon-McIntosh, we show that $g_{2}(\omega ; q)$ and $g_{3}(\omega ; q)$ are, up to classical theta functions and $\eta$-products, the characters of Kac and Wakimoto. As a consequence, we include a "dictionary" that gives a character formula for every classical mock theta function of Ramanujan, as well as subsequent natural generalizations.
\end{abstract}

\section{Introduction AND STATEMENT OF RESUlts}

"Monstrous moonshine" is one of the most revered results connecting modular forms and Lie algebras. Conjectured by Conway and Norton [4] in 1979 and proved by Borcherds [1] in 1992, monstrous moonshine relates the Fourier coefficients of the modular $j$-function, the normalized hauptmodul for $\mathrm{SL}_{2}(\mathbb{Z})$, to dimensions of irreducible representations of the Monster. In his proof, Borcherds constructs a generalized Kac-Moody Lie algebra $M$, and reveals the connection to the $j$-function via a denominator identity satisfied by $M$.

Preceding Moonshine, however, is the fundamental work of V. Kac [6, who first exhibits a link between infinite-dimensional Lie algebras and modular forms. In [6], Kac proves the so-called Weyl-Kac character formula and denominator identity, and interprets the latter in the special case of affine Lie algebras as "Macdonald identities". The key idea that combinatorial identities can be obtained by comparing two different calculations of characters of representations of infinite-dimensional Lie algebras was introduced by Kac in this paper.

Another example relating modular forms and Lie algebras is the fundamental work of Lepowsky and Milne [12, 13, 1978. Here, Lepowsky and Milne relate the Rogers-Ramanujan functions, essentially modular forms of weight 0 , to Weyl-Kac Lie algebras. More specifically, as a consequence of [12, Theorem 5.1], Lepowsky and Milne show that the Rogers-Ramanujan identities, one of which is the following

Received by the editors April 21, 2009 and, in revised form, May 4, 2009.

2000 Mathematics Subject Classification. Primary 11F22, 11F37, 17B67, 11 F50.

(C)2010 American Mathematical Society 
identity between a basic hypergeometric-type series and an infinite product:

$$
\sum_{n \geq 0} q^{n^{2}}(q ; q)_{n}^{-1}=\prod_{n \geq 1}\left(1-q^{5 n-1}\right)^{-1}\left(1-q^{5 n-4}\right)^{-1},
$$

where $(a ; q)_{n}:=\prod_{j=0}^{n-1}\left(1-a q^{j}\right)$, become principally specialized characters for the standard modules for $A_{1}^{(1)}$, an infinite-dimensional generalized Cartan matrix Lie algebra. In subsequent work in 1981, Lepowsky and Wilson [14 formulate an "abstract" Rogers-Ramanujan identity, which in special cases coincides with the classical identities. There are many other examples of this phenomenon, for example the Virasoro algebras, as well as certain classical infinite-dimensional affine Lie algebras. (See for example the book of Kac 7].)

The more recent work of Kac and Wakimoto [9, 10, 18, beginning in the mid1990s has also made significant connections between number theory and affine Lie superalgebras. Of note along our line of discussion thus far is the 2000 work of D. Zagier [19], who reformulates and proves, in terms of combinatorial modular forms, a conjectured denominator identity of Kac and Wakimoto. Another object of study in the work of Kac and Wakimoto is the specialized character formula for $\operatorname{tr}_{L\left(\Lambda_{(s)} ; r+1\right)} q^{L_{0}}$, where $L\left(\Lambda_{(s)} ; r+1\right)$ is the irreducible $s \ell(r+1,1)^{\wedge}$ module with highest weight $\Lambda_{(s)}$, and $L_{0}$ the "energy operator", or Hamiltonian. In [3], Bringmann and Ono answer a question of Kac regarding the "modularity" of the character formulas $\operatorname{tr}_{L\left(\Lambda_{(s)} ; r+1\right)} q^{L_{0}}$, and show that these character formulas are the "holomorphic parts" of nonholomorphic modular functions.

In addition to exhibiting modular properties, many $q$-series arising from such Lie algebras also have combinatorial properties. For example, the left hand side of the first Rogers-Ramanujan identity (1.1) may be interpreted as the combinatorial generating function for the number of partitions of integers with minimal difference 2 , and the right hand side of (1.1) the generating function for partitions whose parts are congruent to 1 or $4 \bmod 5$. This type of result also holds more generally with respect to Virasoro algebras. Namely, one finds in the work of Kac and Wakimoto [8] and Rocha-Caridi [17] that the generating function for partitions whose parts satisfy congruence relations (an infinite product analogous to the right hand side of (1.1) ) is equal to a character formula:

$$
\operatorname{ch}_{i, k}(q)=q^{\left(h_{i, k}-\frac{c_{k}}{24}\right)} \cdot \prod_{1 \leq n \neq 0, \pm i(\bmod 2 k+1)}\left(1-q^{n}\right)^{-1} .
$$

In light of these results, Ono speculated that the character formulas of Kac and Wakimoto are related to the universal mock theta functions of Gordon and McIntosh. We show that this is indeed the case.

The universal mock theta functions $g_{2}(\omega ; q)$ and $g_{3}(\omega ; q)$ of Gordon and McIntosh [5] are generalizations of the original mock theta functions of Ramanujan. The original mock theta functions are basic hypergeometric-type series appearing in Ramanujan's notebooks and his last letter to Hardy. The functions $g_{2}(\omega ; q)$ and $g_{3}(\omega ; q)$ are aptly named "universal", as it is shown by Gordon and McIntosh [5] that all classical mock theta functions of Ramanujan and subsequent natural generalizations may be written in terms of either $g_{2}(\omega ; q)$ or $g_{3}(\omega ; q)$, depending on the parity of their "order", a number assigned to each mock theta function. Both classically and in their more modern generalizations, mock theta functions have been objects of extensive research in the areas of number theory, combinatorics, 
and mathematical physics. We refer the reader to [16] and [5] for a more detailed account. The universal mock theta functions are defined by

$$
\begin{aligned}
g_{2}(\omega ; q) & :=\sum_{n \geq 0} \frac{(-q ; q)_{n} q^{\frac{1}{2}\left(n^{2}+n\right)}}{(\omega ; q)_{n+1}\left(q \omega^{-1} ; q\right)_{n+1}}, \\
g_{3}(\omega ; q) & :=\sum_{n \geq 0} \frac{q^{n^{2}+n}}{(\omega ; q)_{n+1}\left(q \omega^{-1} ; q\right)_{n+1}} .
\end{aligned}
$$

Kang, in 11], considers the universal mock theta functions $g_{2}(\omega ; q)$ and $g_{3}(\omega ; q)$, and shows that both may be expressed in terms of the "mock Jacobi forms" $\mu(u, v ; \tau)$ studied by Zwegers in his important Ph.D. thesis [20] under the direction of D. Zagier. For a precise definition of $\mu(u, v ; \tau)$ and more on the work and results of Kang and Zwegers, see \$2 below. In addition to results of Bringmann and Ono [3] and Gordon and McIntosh [5], we will use the work of Kang [11] and Zwegers [20] in the course of the proof of our main theorem, Theorem 1.1] below.

Theorem 1.1. Let $q:=e^{2 \pi i \tau}, \tau \in \mathbb{H}$. The following are true.

(i) For $r \in \mathbb{N}$ and $s \in \mathbb{Z}$ we have

$$
g_{2}\left(q^{\frac{r}{4}-\frac{s}{2}} ; q^{\frac{r}{2}}\right)=\widehat{\Theta}_{r}^{-1}(\tau) \operatorname{tr}_{L\left(\Lambda_{(s)} ; r+1\right)} q^{L_{0}}-\widehat{\eta}_{r, s}(\tau) .
$$

(ii) For $r \in 3 \mathbb{N}$ and $s \in \mathbb{Z}$ we have

$$
\begin{aligned}
g_{3} & \left(q^{\frac{r}{9}-\frac{s}{3}} ; q^{\frac{r}{3}}\right) \\
& =\widehat{\Theta}_{r}^{-1}(\tau)\left(q^{\frac{r}{36}+\frac{s}{6}} \cdot \operatorname{tr}_{L\left(\Lambda_{(s)} ; r+1\right)} q^{L_{0}}+q^{-\frac{r}{36}-\frac{s}{6}} \cdot \operatorname{tr}_{L\left(\Lambda_{\left(s+\frac{r}{3}\right)} ; r+1\right)} q^{L_{0}}\right)-\widehat{\beta}_{r, s}(\tau) .
\end{aligned}
$$

(iii) For $r \in 6 \mathbb{N}$ and $s \equiv \frac{r}{6}(\bmod r)$ we have

$$
g_{3}\left(q^{\frac{r}{9}-\frac{s}{3}} ; q^{\frac{r}{3}}\right)=\widehat{\Theta}_{r}^{-1}(\tau) \cdot q^{\frac{r}{36}+\frac{s}{6}} \cdot \operatorname{tr}_{L\left(\Lambda_{(s)} ; r+1\right)} q^{L_{0}}-\widehat{\psi}_{r, s}(\tau) .
$$

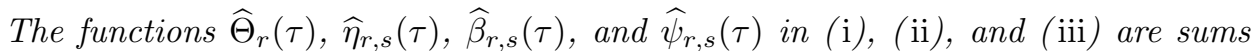
of modular forms defined in (2.14), (2.15), (2.20) and (2.24).

By subtracting the identity in Theorem 1.1(iii) from that in (iii), we see that with hypotheses on $r$ and $s$ the Kac-Wakimoto character formula is a sum of modular forms.

Corollary 1.2. Let $r \in 6 \mathbb{N}$ and $s \equiv \frac{r}{6}(\bmod r)$. Then we have

$$
\left.\widehat{\Theta}_{r}^{-1}(\tau) \cdot q^{-\frac{r}{36}-\frac{s}{6}} \cdot \operatorname{tr}_{L(\Lambda}\left(s+\frac{r}{3}\right) ; r+1\right) q^{L_{0}}=\widehat{\beta}_{r, s}(\tau)-\widehat{\psi}_{r, s}(\tau) .
$$

Example 1. The third order mock theta function of Ramanujan,

$$
f(q):=\sum_{n \geq 0} \frac{q^{n^{2}}}{(-q ; q)_{n}^{2}},
$$

has been of particular interest. Notable recent works include those of Bringmann and Ono [2] and Zwegers [20. Using formula (3.11) of [5] which relates $f(q)$ to the universal mock theta function $g_{3}(\omega ; q)$, we find, after making appropriate substitutions in Theorem 1.1 (iii), that the third order mock theta function $f(q)$ can be 
expressed in terms of the Kac-Wakimoto character formulas as follows:

$$
f(-q)=-4 q \widehat{\Theta}_{12}^{-1}(\tau)\left(q^{\frac{1}{2}} \cdot \operatorname{tr}_{L\left(\Lambda_{(1), 13}\right)} q^{L_{0}}+q^{-\frac{1}{2}} \cdot \operatorname{tr}_{L\left(\Lambda_{(5)} ; 13\right)} q^{L_{0}}\right)+F(\tau) .
$$

Here, $F(\tau)$ is a sum of modular forms defined by

$$
F(\tau):=4 q \widehat{\beta}_{12,1}(\tau)+\frac{\left(q^{2} ; q^{2}\right)_{\infty}^{7}}{(q ; q)_{\infty}^{3}\left(q^{4} ; q^{4}\right)_{\infty}^{3}} .
$$

Here and throughout, $(a ; q)_{\infty}:=\lim _{n \rightarrow \infty}(a ; q)_{n}$.

Example 2. As a second example, we consider the order 10 mock theta function appearing in Ramanujan's lost notebook:

$$
\phi(q):=\sum_{n \geq 0} \frac{q^{\frac{1}{2} n(n+1)}}{\left(q ; q^{2}\right)_{n+1}} .
$$

Using the expression for $\phi(q)$ in terms of the universal mock theta function $g_{2}(\omega ; q)$ found in [5, (5.18)] and applying Theorem 1.1 (1i), we find

$$
\phi(q)=2 q \cdot \widehat{\Theta}_{10}^{-1}(\tau) \cdot \operatorname{tr}_{L\left(\Lambda_{(1)} ; 11\right)} q^{L_{0}}+\Phi(\tau),
$$

where

$$
\Phi(\tau):=i q^{\frac{5}{8}} \cdot \frac{\left(q^{10} ; q^{10}\right)_{\infty}^{2}}{\left(q^{5} ; q^{5}\right)_{\infty}} \cdot \frac{\vartheta\left(2 \tau+\frac{1}{2} ; 5 \tau\right)}{\vartheta(2 \tau ; 10 \tau)}-2 q \cdot \widehat{\eta}_{10,1}(\tau)
$$

Remark 1. In the Appendix (\$3), we provide a "dictionary" that gives a character formula for every classical mock theta function of Ramanujan, as well as subsequent natural generalizations. The identities are derived from Theorem 1.1 in a manner similar to that described in Example 1 and Example 2.

\section{Proof of Theorem 1.1}

In this section we will prove Theorem 1.1. In the course of our proof we will make use of results due to Bringmann and Ono 3 in their study of the KacWakimoto character formulas $\operatorname{tr}_{L\left(\Lambda_{(s)} ; r+1\right)} q^{L_{0}}$, results due to Gordon-McIntosh [5] on the universal mock theta functions, work of Kang [11] who relates the universal mock theta functions to Zwegers' mock Jacobi forms $\mu(u, v ; \tau)$, as well as the work of Zwegers [20] on the mock Jacobi forms $\mu(u, v ; \tau)$. $\mathbb{Z}$

We begin with the work of Kac and Wakimoto [10], which implies for $r \in \mathbb{N}, s \in$

$$
\operatorname{tr}_{L\left(\Lambda_{(s)} ; r+1\right)} q^{L_{0}}=2 q^{\frac{r-1}{24}-\frac{s}{2}} \cdot \frac{\eta^{2}(2 \tau)}{\eta^{r+3}(\tau)} \cdot L_{r, s}(\tau)
$$

where

$$
L_{r, s}(\tau):=\sum_{k=\left(k_{1}, k_{2}, \ldots, k_{r}\right) \in \mathbb{Z}^{r}} \frac{q^{\frac{1}{2} \sum_{i} k_{i}\left(k_{i}+1\right)}}{1+q^{-s+\sum_{i} k_{i}}} .
$$

In [3, Bringmann and Ono explicitly relate the character formulas in (2.1) to weight 0 nonholomorphic forms. A key identity used in their work, which we will also make use of here, is the following [3, Theorem 2.1]. 
Proposition (Bringmann-Ono). For integers $r \geq 1$ and $s \in \mathbb{Z}$, we have

$$
L_{r, s}(\tau)=\sum_{d=\left(d_{1}, d_{2}, \ldots, d_{r}\right) \in D_{r}} \widetilde{\vartheta}_{d}(\tau) \mu_{d_{r}, r, s}(\tau) .
$$

The sum in (2.2) is a finite lattice sum over $D_{r}:=\left\{d=\left(d_{1}, d_{2}, \ldots, d_{r}\right) \in \mathbb{Z}^{r} \mid 0 \leq\right.$ $d_{i} \leq i-1$ for each $\left.1 \leq i \leq r\right\}$. The functions $\widetilde{\vartheta}_{d}(\tau)$ and $\mu_{d_{r}, r, s}(\tau)$ are defined by

$$
\begin{aligned}
\widetilde{\vartheta}_{d}(\tau) & :=\prod_{i=1}^{r-1} \vartheta\left(d_{i},-d_{i+1}, i, i+1 ; \tau\right), \\
\mu_{d_{r}, r, s}(\tau) & :=q^{-\frac{d_{r}}{2}+\frac{d_{r}^{2}}{2 r}} \sum_{n \in \mathbb{Z}} \frac{q^{\frac{r n}{2}(n+1)-d n}}{1+q^{r n-d-s}},
\end{aligned}
$$

where

$$
\vartheta(a, b, c, d ; \tau):=q^{\frac{c d}{2}\left(\frac{a}{c}+\frac{b}{d}-\frac{1}{2}\right)^{2}} \vartheta\left(\left(a d+b c-\frac{c d}{2}\right) \tau-\frac{1}{2} ; c d \tau\right),
$$

and the function $\vartheta(z ; \tau)$ defining (2.5) is the classical Jacobi theta function

$$
\vartheta(z ; \tau):=\sum_{n \in \mathbb{Z}} e^{\pi i\left(n+\frac{1}{2}\right)^{2} \tau+2 \pi i\left(n+\frac{1}{2}\right)\left(z+\frac{1}{2}\right)} .
$$

(The empty product in (2.3) is understood to equal 1.) One also understands the modularity of the function $\mu_{d_{r}, r, s}(\tau)$. An observation of Bringmann and Ono in [3] reveals that

$$
q^{-\frac{s}{2}} \mu_{d_{r}, r, s}(\tau)=-i q^{\frac{d_{r}^{2}}{2 r}} \vartheta\left(\frac{1}{2}-d_{r} \tau ; r \tau\right) \mu\left(\frac{1}{2}-\left(s+d_{r}\right) \tau, \frac{1}{2}-d_{r} \tau ; r \tau\right),
$$

where the function $\mu(u, v ; \tau)$ is nearly a weight $1 / 2$ Jacobi form studied by Zwegers in [20, who shows that a correction term may be added to the function $\mu(u, v ; \tau)$ to produce weight $1 / 2$ harmonic weak Maass forms at torsion points. The function $\mu(u, v ; \tau)$ is defined by

$$
\mu(u, v ; \tau):=\frac{e(u / 2)}{\vartheta(v ; \tau)} \sum_{n \in \mathbb{Z}} \frac{(-1)^{n} e(n v) q^{n(n+1) / 2}}{1-e(u) q^{n}},
$$

where $e(\alpha):=e^{2 \pi i \alpha}$. Of the fundamental properties and transformations associated to $\mu(u, v ; \tau)$ (given in [20]), we will make use of the following.

Proposition (Zwegers). Let $u, v, u+z, v+z \in \mathbb{C} \backslash \mathbb{Z} \tau+\mathbb{Z}, \tau \in \mathbb{H}$. Then

$$
\mu(u+z, v+z ; \tau)=\mu(u, v ; \tau)+\frac{i \eta^{3}(\tau) \vartheta(u+v+z ; \tau) \vartheta(z ; \tau)}{\vartheta(u ; \tau) \vartheta(v ; \tau) \vartheta(u+z ; \tau) \vartheta(v+z ; \tau)} .
$$

The function $\eta(\tau)$ in (2.8) is the Dedekind $\eta$-function, a classical weight $1 / 2$ modular form defined by

$$
\eta(\tau):=q^{\frac{1}{24}} \prod_{n=1}^{\infty}\left(1-q^{n}\right)
$$


Recalling (2.2) and (2.7), we begin by applying (2.8) with

$$
\begin{aligned}
u & :=\left(\frac{r}{2}-s\right) \tau, \\
v & :=\frac{r \tau}{2}, \\
z & :=\frac{1}{2}-\left(d_{r}+\frac{r}{2}\right) \tau
\end{aligned}
$$

to the $\mu$-function appearing in (2.7), and find

$$
\mu\left(\frac{1}{2}-\left(s+d_{r}\right) \tau, \frac{1}{2}-d_{r} \tau ; r \tau\right)=\mu\left(\left(\frac{r}{2}-s\right) \tau, \frac{r \tau}{2} ; r \tau\right)+\psi_{r, s, d_{r}}(\tau),
$$

where

$$
\psi_{r, s, d_{r}}(\tau):=\frac{i \eta^{3}(r \tau) \vartheta\left(\frac{1}{2}+\left(\frac{r}{2}-s-d_{r}\right) \tau ; r \tau\right) \vartheta\left(\frac{1}{2}-\left(d_{r}+\frac{r}{2}\right) \tau ; r \tau\right)}{\vartheta\left(\left(\frac{r}{2}-s\right) \tau ; r \tau\right) \vartheta\left(\frac{r \tau}{2} ; r \tau\right) \vartheta\left(\frac{1}{2}-\left(s+d_{r}\right) \tau ; r \tau\right) \vartheta\left(\frac{1}{2}-d_{r} \tau ; r \tau\right)} .
$$

Next, we let

$$
\mathcal{S}_{1}(r, s ; \tau):=-i q^{\frac{s}{2}} \sum_{\left(d_{1}, \ldots, d_{r}\right) \in D_{r}} q^{\frac{d_{r}^{2}}{2 r}} \vartheta\left(\frac{1}{2}-d_{r} \tau ; r \tau\right) \widetilde{\vartheta}_{d}(\tau) \mu\left(\left(\frac{r}{2}-s\right) \tau, \frac{r \tau}{2} ; r \tau\right),
$$

$$
\mathcal{S}_{2}(r, s ; \tau):=-i q^{\frac{s}{2}} \sum_{\left(d_{1}, \ldots, d_{r}\right) \in D_{r}} q^{\frac{d_{r}^{2}}{2 r}} \vartheta\left(\frac{1}{2}-d_{r} \tau ; r \tau\right) \widetilde{\vartheta}_{d}(\tau) \psi_{r, s, d_{r}}(\tau) .
$$

By (2.2) and (2.9) we have

$$
L_{r, s}(\tau)=\mathcal{S}_{1}(r, s ; \tau)+\mathcal{S}_{2}(r, s ; \tau) .
$$

Next, we define

$$
\begin{aligned}
& \widehat{\Theta}_{r}(\tau):=2 q^{\frac{r}{6}-\frac{1}{24} \Theta_{r}(\tau),} \\
& \widehat{\eta}_{r, s}(\tau):=i q^{-\frac{r}{4}+\frac{s}{2}} \cdot \frac{\eta^{4}(r \tau)}{\eta^{2}\left(\frac{r \tau}{2}\right) \vartheta\left(\left(\frac{r}{2}-s\right) \tau ; r \tau\right)} \\
& \qquad \begin{array}{ll}
-\frac{q^{-\frac{r}{8}}}{2} \cdot \frac{\eta^{4}(r \tau)}{\eta^{2}(2 r \tau)} \cdot \frac{\vartheta\left(\frac{1}{2}-\frac{r \tau}{2} ; r \tau\right) \vartheta\left(\frac{1}{2}+\left(\frac{r}{2}-s\right) \tau ; r \tau\right)}{\vartheta\left(\frac{1}{2}-s \tau ; r \tau\right) \vartheta\left(\left(\frac{r}{2}-s\right) \tau ; r \tau\right) \vartheta\left(\frac{r \tau}{2} ; r \tau\right)}, & \frac{s}{r} \in \frac{1}{2}+\mathbb{Z}, \\
+q^{-\frac{s}{2}-\frac{r}{8}} \cdot \Theta_{r}^{-1}(\tau) \frac{\eta^{2}(2 \tau)}{\eta^{r+3}(\tau)} \cdot \mathcal{S}_{2}(r, s ; \tau), & \frac{s}{r} \notin \frac{1}{2}+\mathbb{Z},
\end{array}
\end{aligned}
$$

where the function $\Theta_{r}(\tau)$ is the weight $-1 / 2$ modular form defined in [3] by

$$
\Theta_{r}(\tau):=\frac{\eta^{2}(2 \tau)}{\eta^{r+3}(\tau)} \cdot \sum_{d=\left(d_{1}, d_{2}, \ldots, d_{r}\right) \in D_{r}} \tilde{\vartheta}_{d}(\tau) \cdot q^{\frac{d_{r}^{2}}{2 r}} \vartheta\left(\frac{1}{2}-d_{r} \tau ; r \tau\right),
$$

and $\mathcal{S}_{2}(r, s ; \tau)$ is the finite sum of modular forms defined in (2.12). We note that in 3 it is pointed out that

$$
\Theta_{r}(\tau)=-2^{r} \frac{\eta^{2 r+2}(2 \tau)}{\eta^{2 r+3}(\tau)}
$$

Proof of Theorem 1.1 (1). The proof of Theorem 1.1 (1i) will now follow from Proposition 2.1 below, after a straightforward calculation using (2.1), (2.2), (2.12), (2.13), (2.14) and (2.15). 
Proposition 2.1. For $r \in \mathbb{N}$ and $s \in \mathbb{Z}$, we have that

$$
\begin{aligned}
\mathcal{S}_{1}(r, s ; \tau) & =q^{\frac{r}{8}+\frac{s}{2}} \cdot \frac{\eta^{r+3}(\tau)}{\eta^{2}(2 \tau)} \cdot \Theta_{r}(\tau) \cdot g_{2}\left(q^{\frac{r}{4}-\frac{s}{2}} ; q^{\frac{r}{2}}\right) \\
& +i q^{s-\frac{r}{8}} \cdot \Theta_{r}(\tau) \cdot \frac{\eta^{4}(r \tau) \eta^{r+3}(\tau)}{\eta^{2}(2 \tau) \eta^{2}\left(\frac{r \tau}{2}\right) \vartheta\left(\left(\frac{r}{2}-s\right) \tau ; r \tau\right)},
\end{aligned}
$$

and for $r \in \mathbb{N}, s \in \mathbb{Z}$ such that $\frac{s}{r} \in \frac{1}{2}+\mathbb{Z}$, we have that

$$
\begin{aligned}
\mathcal{S}_{2}(r, s ; \tau)=-\frac{q^{\frac{s}{2}}}{2} & \cdot \frac{\eta^{4}(r \tau) \eta^{r+3}(\tau)}{\eta^{2}(2 \tau) \eta^{2}(2 r \tau)} \cdot \frac{\vartheta\left(\frac{1}{2}-\frac{r \tau}{2} ; r \tau\right) \vartheta\left(\frac{1}{2}+\left(\frac{r}{2}-s\right) \tau ; r \tau\right)}{\vartheta\left(\frac{1}{2}-s \tau ; r \tau\right) \vartheta\left(\left(\frac{r}{2}-s\right) \tau ; r \tau\right) \vartheta\left(\frac{r \tau}{2} ; r \tau\right)} \\
& \cdot \Theta_{r}(\tau) .
\end{aligned}
$$

Proof of Theorem 1.1 (iii). To prove Theorem 1.1(iii), we use the following relation between the universal mock theta functions $g_{2}(\omega ; q)$ and $g_{3}(\omega ; q)$ due to Gordon and McIntosh [5].

Proposition (Gordon-McIntosh). The following identity is true:

$$
g_{3}\left(\zeta^{4} ; q^{4}\right)=q \zeta^{-2} g_{2}\left(\zeta^{6} q ; q^{6}\right)+q^{-1} \zeta^{2} g_{2}\left(\zeta^{6} q^{-1} ; q^{6}\right)-J(\zeta ; q),
$$

where $\zeta=e^{2 \pi i z}$, and

$$
J(\zeta ; q):=\zeta^{4} q^{\frac{7}{4}} \cdot \frac{\left(q^{2} ; q^{2}\right)_{\infty}^{3}\left(q^{12} ; q^{12}\right)_{\infty}}{\left(q^{4} ; q^{4}\right)_{\infty}\left(q^{6} ; q^{6}\right)_{\infty}^{2}} \cdot \frac{\vartheta(2 z+\tau ; 2 \tau) \vartheta(12 z+6 \tau ; 12 \tau)}{\vartheta(4 z ; 2 \tau) \vartheta(6 z-\tau ; 2 \tau)} .
$$

Next we define

$$
\widehat{\beta}_{r, s}(\tau):=J\left(q^{\frac{r}{36}-\frac{s}{12}} ; q^{\frac{r}{12}}\right)+q^{\frac{r}{36}+\frac{s}{6}} \cdot \widehat{\eta}_{r, s}(\tau)+q^{-\frac{r}{36}-\frac{s}{6}} \widehat{\eta}_{r, s+\frac{r}{3}}(\tau) .
$$

In (2.19), we replace $q$ by $q^{\frac{r}{12}}$ and then let $\zeta=q^{\frac{r}{36}-\frac{s}{12}}$. With these substitutions, (2.19) becomes

$$
\begin{aligned}
g_{3}\left(q^{\frac{r}{9}-\frac{s}{3}} ; q^{\frac{r}{3}}\right) & =q^{\frac{r}{36}+\frac{s}{6}} \cdot g_{2}\left(q^{\frac{r}{4}-\frac{s}{2}} ; q^{\frac{r}{2}}\right)+q^{-\frac{r}{36}-\frac{s}{6}} \cdot g_{2}\left(q^{\frac{r}{4}-\frac{s+\frac{r}{3}}{2}} ; q^{\frac{r}{2}}\right) \\
& -J\left(q^{\frac{r}{36}-\frac{s}{12}} ; q^{\frac{r}{12}}\right) .
\end{aligned}
$$

Theorem 1.1(iii) will now follow after a short calculation from Theorem 1.1(ii) using (2.21) and (2.20).

Proof of Theorem 1.1 (iii). From the work of Gordon and McIntosh [5], one can also deduce another relation between the universal mock theta functions $g_{2}\left(\omega_{1} ; q\right)$ and $g_{3}\left(\omega_{2} ; q\right)$ under suitable hypotheses on the arguments $\omega_{1}$ and $\omega_{2}$. Namely, from [5. Equation (7.1)] and the expressions given for the functions $u_{2}$ and $u_{3}$ following (6.9) on page 37 of [5], one may deduce the following:

$$
g_{3}\left(x(q)^{4} ; q^{4}\right)=-i q^{-\frac{1}{2}} \frac{T(x(q), q)}{\vartheta(4 \tau ; 12 \tau)}+q x(q)^{-2} g_{2}\left(x(q)^{6} q ; q^{6}\right),
$$

where $x(q)=q^{\frac{1}{6}+n}, n \in \mathbb{Z}$, and $T(x(q), q)$ is a theta function defined in [15.

To prove (iii), we first replace $q$ by $q^{\frac{r}{12}}$ in (2.22). With this change of variable, we see that $x(q)^{6} q$ is replaced by $q^{\frac{r}{6}+\frac{r n}{2}}$, and $q^{6}$ is replaced by $q^{\frac{r}{2}}$. Thus, with these substitutions, we find the universal mock theta function $g_{2}\left(q^{\frac{r}{6}+\frac{r n}{2}} ; q^{\frac{r}{2}}\right)$ in (2.22). 
If we set $\frac{r}{6}+\frac{r n}{2}=\frac{r}{4}-\frac{s}{2}$, we see that because we require $s, n \in \mathbb{Z}$ and $r \in \mathbb{N}$, we must have $r \in 6 \mathbb{N}$ and $s \equiv \frac{r}{6}(\bmod r)$. Combining these observations, we deduce for $r \in 6 \mathbb{N}$ and $s \equiv \frac{r}{6}(\bmod r)$ that

$$
g_{3}\left(q^{\frac{r}{9}-\frac{s}{3}} ; q^{\frac{r}{3}}\right)=q^{\frac{r}{36}+\frac{s}{6}} g_{2}\left(q^{\frac{r}{4}-\frac{s}{2}} ; q^{\frac{r}{2}}\right)-i q^{-\frac{r}{24}} \frac{T\left(q^{\frac{r}{36}-\frac{s}{12}}, q^{\frac{r}{12}}\right)}{\vartheta\left(\frac{r \tau}{3} ; r \tau\right)} .
$$

Next we define

$$
\widehat{\psi}_{r, s}(\tau):=i q^{-\frac{r}{24}} \frac{T\left(q^{\frac{r}{36}-\frac{s}{12}}, q^{\frac{r}{12}}\right)}{\vartheta\left(\frac{r \tau}{3} ; r \tau\right)}+q^{\frac{r}{36}+\frac{s}{6}} \widehat{\eta}_{r, s}(\tau) .
$$

Theorem 1.1 (iii) now follows after combining (2.23) with Theorem 1.1 (ii).

Proof of Proposition 2.1 (2.17). We observe that (2.17) will follow after applying to the function $\mathcal{S}_{1}(r, s ; \tau)$ defined in (2.11) the following proposition due to Kang [11, which relates the universal mock theta function $g_{2}(\omega ; q)$ to Zwegers' functions $\mu(u, v ; \tau)$.

Proposition (Kang). Let $\alpha \in \mathbb{C}$ be such that $\alpha \notin \mathbb{Z}+2 \tau \mathbb{Z}$. Then

$$
i e(\alpha) g_{2}(e(\alpha) ; q)=\frac{\eta^{4}(2 \tau)}{\eta^{2}(\tau) \vartheta(2 \alpha ; 2 \tau)}+e(\alpha) q^{-\frac{1}{4}} \mu(2 \alpha, \tau ; 2 \tau) .
$$

More specifially, one lets $\alpha=\frac{1}{2}\left(\frac{r}{2}-s\right) \tau$ in (2.25), and replaces $\tau$ by $r \tau / 2$. The identity given in (2.17) follows after a short calculation.

Proof of Proposition 2.1 (2.18). The proof of (2.18) requires more work. We first observe that many of the functions defining $\mathcal{S}_{2}(r, s ; \tau)$ are independent of the indices of summation $d=\left(d_{1}, d_{2}, \ldots, d_{r}\right) \in D_{r}$. To this end we define

$$
\widehat{\mathcal{S}}_{2}(r, s ; \tau):=\sum_{d \in D_{r}} q^{\frac{d_{r}^{2}}{2 r}} \widetilde{\vartheta}_{d}(\tau) \frac{\vartheta\left(\frac{1}{2}+\left(\frac{r}{2}-s-d_{r}\right) \tau ; r \tau\right) \vartheta\left(\frac{1}{2}-\left(d_{r}+\frac{r}{2}\right) \tau ; r \tau\right)}{\vartheta\left(\frac{1}{2}-\left(s+d_{r}\right) \tau ; r \tau\right)}
$$

so that

$$
\mathcal{S}_{2}(r, s ; \tau)=\frac{q^{\frac{s}{2}} \eta^{3}(r \tau)}{\vartheta\left(\left(\frac{r}{2}-s\right) \tau ; r \tau\right) \vartheta\left(\frac{r \tau}{2} ; r \tau\right)} \cdot \widehat{\mathcal{S}}_{2}(r, s ; \tau) .
$$

Here, our goal is to rewrite the $\operatorname{sum} \widehat{\mathcal{S}}_{2}(r, s ; \tau)$, not as a finite lattice sum over elements $\left(d_{1}, d_{2}, \ldots, d_{r}\right) \in D_{r}$, but as a visible product of modular forms. We begin by using the well-known product expansion for the Jacobi theta functions $\vartheta(\omega ; \tau)$, known as the Jacobi triple product identity:

$$
\vartheta(\omega ; \tau)=-i q^{\frac{1}{8}} e^{-\pi i \omega} \prod_{n \geq 1}\left(1-q^{n}\right)\left(1-e(\omega) q^{n-1}\right)\left(1-e(-\omega) q^{n}\right) .
$$

Next, we let

$$
\begin{aligned}
& F_{r, s}(q, z) \\
& :=\prod_{n \geq 1} \frac{\left(1+q^{r(n-1)+\frac{r}{2}-s} z\right)\left(1+q^{r n-\frac{r}{2}+s} z^{-1}\right)\left(1+q^{r(n-1)-\frac{r}{2}} z\right)\left(1+q^{r n+\frac{r}{2}} z^{-1}\right)}{\left(1+q^{r(n-1)-s} z\right)\left(1+q^{r n+s} z^{-1}\right)},
\end{aligned}
$$


so that after applying (2.28) to (2.26), we have

$$
\widehat{\mathcal{S}}_{2}(r, s ; \tau)=-q^{\frac{r}{8}} \prod_{n \geq 1}\left(1-q^{r n}\right) \sum_{d=\left(d_{1}, d_{2}, \ldots, d_{r}\right) \in D_{r}} q^{\frac{d_{r}^{2}}{2 r}+\frac{d_{r}}{2}} \widetilde{\vartheta}_{d}(\tau) F_{r, s}\left(q, q^{-d_{r}}\right)
$$

We next establish the following series expansion for the function $F_{r, s}(q, z)$.

Lemma 2.2. For $r \in \mathbb{N}, s \in \mathbb{Z}$ such that $\frac{s}{r} \in \frac{1}{2}+\mathbb{Z}$, the function $F_{r, s}(q, z)$ satisfies

$$
F_{r, s}(q, z)=-\frac{q^{\frac{r}{24}}}{2} \cdot \frac{\vartheta\left(\frac{1}{2}+\left(\frac{r}{2}-s\right) \tau ; r \tau\right) \vartheta\left(\frac{1}{2}-\frac{r \tau}{2} ; r \tau\right)}{\vartheta\left(\frac{1}{2}-s \tau ; r \tau\right)} \cdot \frac{1}{\eta^{2}(2 r \tau)} \sum_{n \in \mathbb{Z}} q^{\frac{r n(n-1)}{2}} z^{n} .
$$

Proof. We first observe that

$$
\begin{aligned}
& F_{r, s}\left(q, z q^{r}\right) \\
& =\prod_{n \geq 1} \frac{\left(1+q^{r n+\frac{r}{2}-s} z\right)\left(1+q^{r(n-1)-\frac{r}{2}+s} z^{-1}\right)\left(1+q^{r n-\frac{r}{2}} z\right)\left(1+q^{r(n-1)+\frac{r}{2}} z^{-1}\right)}{\left(1+q^{r n-s} z\right)\left(1+q^{r(n-1)+s} z^{-1}\right)} \\
& =\prod_{n \geq 2} \frac{\left(1+q^{r(n-1)+\frac{r}{2}-s} z\right)\left(1+q^{r(n-1)-\frac{r}{2}} z\right)}{\left(1+q^{r(n-1)-s} z\right)} \prod_{n \geq 0} \frac{\left(1+q^{r n-\frac{r}{2}+s} z^{-1}\right)\left(1+q^{r n+\frac{r}{2}} z^{-1}\right)}{\left(1+q^{r n+s} z^{-1}\right)} \\
& =\frac{\left(1+q^{-s} z\right)\left(1+q^{-\frac{r}{2}+s} z^{-1}\right)\left(1+q^{\frac{r}{2}} z^{-1}\right)}{\left(1+q^{\frac{r}{2}-s} z\right)\left(1+q^{-\frac{r}{2}} z\right)\left(1+q^{s} z^{-1}\right)} F_{r, s}(q, z) \\
& =z^{-1} F_{r, s}(q, z) .
\end{aligned}
$$

Next, we wish to use the relation (2.32) to write a power series expansion for $F_{r, s}(q, z)$ in the variable $z$. We note that $F_{r, s}(q, z)$ as a function in $z$ has poles for $z=-q^{r n+s}, n \in \mathbb{Z}$. By making the assumption that $\frac{s}{r} \in \frac{1}{2}+\mathbb{Z}$, we see that for each pole there is a corresponding zero of the same order, and thus $F_{r, s}(q, z)$ is holomorphic. For example, if $\frac{s}{r} \in \frac{1}{2}+\mathbb{Z}^{\geq 0}$, each of the poles $z=-q^{r n+s}, n \geq 1$, will be canceled by the zero given by $z=-q^{r m_{n}+\frac{r}{2}}$, where $m_{n}:=n+\frac{s}{r}-\frac{1}{2} \geq \frac{1}{2}+\frac{s}{r}$, which is an integer $\geq 1$. One can argue similarly that each of the poles $z=-q^{r n+s}, n \leq 0$, will be canceled by other zeros of the function $F_{r, s}(q, z)$. The case $\frac{s}{r} \in-\frac{1}{2}+\mathbb{Z} \leq 0$ follows by a similar argument. Thus, with these assumptions on $r$ and $s$, if we write the power series expansion for $F_{r, s}(q, z)$ in $z$ as

$$
F_{r, s}(q, z)=\sum_{n \in \mathbb{Z}} a_{n}(q) z^{n}
$$

then the relation (2.32) implies

$$
a_{n}(q) \cdot q^{r n}=a_{n+1}(q)
$$


After iterating (2.34), one finds that $a_{n}(q)=q^{\frac{r n(n-1)}{2}} a_{0}(q)$ for all $n \in \mathbb{Z}$. Thus, we have

$$
F_{r, s}(q, z)=a_{0}(q) \sum_{n \in \mathbb{Z}} q^{\frac{r n(n-1)}{2}} z^{n} .
$$

To determine $a_{0}(q)$, we see using (2.29) that

$$
F_{r, s}(q, 1)=-q^{-\frac{r}{8}+\frac{r}{24}} \frac{\vartheta\left(\frac{1}{2}+\left(\frac{r}{2}-s\right) \tau ; r \tau\right) \vartheta\left(\frac{1}{2}-\frac{r \tau}{2} ; r \tau\right)}{\vartheta\left(\frac{1}{2}-s \tau ; r \tau\right) \eta(r \tau)} .
$$

On the other hand, using (2.35) we see that

$$
\begin{aligned}
F_{r, s}(q, 1) & =q^{-\frac{r}{8}} a_{0}(q) \sum_{n \in \mathbb{Z}} q^{\frac{r}{2}\left(n+\frac{1}{2}\right)^{2}} \\
& =2 a_{0}(q) \prod_{n \geq 1}\left(1-q^{r n}\right)\left(1+q^{r n}\right)^{2},
\end{aligned}
$$

where the product expansion in (2.38) is determined by writing the sum defining (2.37) in terms of the Jacobi theta function $\vartheta(z ; \tau)$, applying (2.28), and simplifying. Combining (2.38) with (2.36) implies

$$
a_{0}(q)=-\frac{q^{\frac{r}{24}}}{2} \cdot \frac{\vartheta\left(\frac{1}{2}+\left(\frac{r}{2}-s\right) \tau ; r \tau\right) \vartheta\left(\frac{1}{2}-\frac{r \tau}{2} ; r \tau\right)}{\vartheta\left(\frac{1}{2}-s \tau ; r \tau\right)} \cdot \frac{1}{\eta^{2}(2 r \tau)} .
$$

Substituting(2.39) into (2.35) finishes the proof.

To continue, we apply the series expansion for $F_{r, s}(q, z)$ obtained in (2.31) to the expression for $\widehat{\mathcal{S}}_{2}(r, s ; \tau)$ given in (2.30), replacing $z$ by $q^{-d}$. Once again, many of the functions and terms appearing are independent of the indices of summation $d=\left(d_{1}, d_{2}, \ldots, d_{r}\right) \in D_{r}$. One finds that the only sum left to be understood (with respect to $\left.\widehat{\mathcal{S}}_{2}(r, s ; \tau)\right)$ is

$$
\sum_{d=\left(d_{1}, d_{2}, \ldots, d_{r}\right) \in D_{r}} q^{\frac{d_{r}^{2}}{2 r}+\frac{d_{r}}{2}} \widetilde{\vartheta}_{d}(\tau) \sum_{n \in \mathbb{Z}} q^{\frac{r n(n-1)}{2}-d_{r} n} .
$$

A short calculation reveals

$$
-q^{-\frac{r}{8}-\frac{d_{r}}{2}} \vartheta\left(\frac{1}{2}-d_{r} \tau ; r \tau\right)=\sum_{n \in \mathbb{Z}} q^{\frac{r n(n-1)}{2}-d_{r} n} .
$$

Applying (2.41) to (2.40) shows that the sum in (2.40) may be written as

$$
-q^{-\frac{r}{8}} \sum_{d=\left(d_{1}, d_{2}, \ldots, d_{r}\right) \in D_{r}} q^{\frac{d_{r}^{2}}{2 r}} \widetilde{\vartheta}_{d}(\tau) \vartheta\left(\frac{1}{2}-d_{r} \tau ; r \tau\right) .
$$

It is explained in 3 that the sum in (2.42) is equal to

$$
-q^{-\frac{r}{8}} \frac{\eta^{r+3}(\tau)}{\eta^{2}(2 \tau)} \Theta_{r}(\tau)
$$

In summary, one begins with the expression in (2.27) for $\mathcal{S}_{2}(r, s ; \tau)$ and substitutes (2.35) into (2.30), using the expression for $a_{0}(q)$ derived above in (2.39). Finally, one substitutes for (2.42) the expression $-q^{-\frac{r}{8}} \frac{\eta^{r+3}(\tau)}{\eta^{2}(2 \tau)} \Theta_{r}(\tau)$. After simplifying, one finds (2.18). 


\section{Appendix: Character formulas for the mock theta functions}

In this section, we give a list of character formulas for all classical mock theta functions as well as subsequent natural generalizations. To derive them, we apply Theorem 1.1 to the "mock theta conjectures". The mock theta conjectures are no longer conjectures, but are now known identities due to G. Andrews, F. Garvan, D. Hickerson, B. Gordon, R. McIntosh and Y.-S. Choi. The conjectures may be expressed as formulas relating each mock theta function to the universal mock theta functions $g_{2}(\omega ; q)$ and $g_{3}(\omega ; q)$. We follow the notation and presentation of the mock theta conjectures as they appear in [5. (3.4), (3.5), (3.10), (3.11), (5.2), (5.10), (5.12), (5.18)], and refer the reader there for a complete account. The auxiliary functions $G(q), H(q), \theta_{4}(0, q), \psi(q)$, and $j(x, q)$ appearing are defined in Table 8 at the end of this section. We remark that there exist mock theta functions other than those listed in Tables 10 - 7. However it is unnecessary to list their character formulas here: they are easily derived using the formulas provided in Tables 1- 7, as well as "linear relations" between these mock theta functions and those listed below. (See [5].) 
TABLE 1. Character formulas for order 3 mock $\theta$-functions

$$
\begin{aligned}
& f(-q)=-4 q \cdot \widehat{\Theta}_{12}^{-1}\left(q^{\frac{1}{2}} \cdot \operatorname{tr}_{L\left(\Lambda_{(1)} ; 13\right)} q^{L_{0}}+q^{-\frac{1}{2}} \cdot \operatorname{tr}_{L\left(\Lambda_{(5)} ; 13\right)} q^{L_{0}}\right) \\
& +\quad 4 q \widehat{\beta}_{12,1}(\tau)+\frac{\left(q^{2} ; q^{2}\right)_{\infty}^{7}}{(q ; q)_{\infty}^{3}\left(q^{4} ; q^{4}\right)_{\infty}^{3}} \\
& \phi(q)=-2 q \cdot \widehat{\Theta}_{12}^{-1}\left(q^{\frac{1}{2}} \cdot \operatorname{tr}_{L\left(\Lambda_{(1)} ; 13\right)} q^{L_{0}}+q^{-\frac{1}{2}} \cdot \operatorname{tr}_{L\left(\Lambda_{(5)} ; 13\right)} q^{L_{0}}\right) \\
& +\quad+2 q \widehat{\beta}_{12,1}(\tau)+\frac{\left(q^{2} ; q^{2}\right)_{\infty}^{7}}{(q ; q)_{\infty}^{3}\left(q^{4} ; q^{4}\right)_{\infty}^{3}} \\
& \psi(q)=q \cdot \widehat{\Theta}_{12}^{-1}\left(q^{\frac{1}{2}} \cdot \operatorname{tr}_{L\left(\Lambda_{(1)} ; 13\right)} q^{L_{0}}+q^{-\frac{1}{2}} \cdot \operatorname{tr}_{L\left(\Lambda_{(5)} ; 13\right)} q^{L_{0}}\right)-q \widehat{\beta}_{12,1}(\tau) \\
& \chi(-q)=-q \cdot \widehat{\Theta}_{12}^{-1}\left(q^{\frac{1}{2}} \cdot \operatorname{tr}_{L\left(\Lambda_{(1)} ; 13\right)} q^{L_{0}}+q^{-\frac{1}{2}} \cdot \operatorname{tr}_{L\left(\Lambda_{(5)} ; 13\right)} q^{L_{0}}\right) \\
& +q \widehat{\beta}_{12,1}(\tau)+\frac{\left(q^{4} ; q^{4}\right)_{\infty}^{3}\left(q^{6} ; q^{6}\right)_{\infty}^{3}}{\left(q^{2} ; q^{2}\right)_{\infty}^{2}\left(q^{3} ; q^{3}\right)_{\infty}\left(q^{12} ; q^{12}\right)_{\infty}^{2}} \\
& \omega(q)=\widehat{\Theta}_{6}^{-1}\left(\operatorname{tr}_{L\left(\Lambda_{(-1)} ; 7\right)} q^{L_{0}}+\operatorname{tr}_{L\left(\Lambda_{(1)} ; 7\right)} q^{L_{0}}\right)-\widehat{\beta}_{6,-1}(\tau) \\
& v(q)=-q \cdot \widehat{\Theta}_{12}^{-1}\left(\operatorname{tr}_{L\left(\Lambda_{(-2)} ; 13\right)} q^{L_{0}}+\operatorname{tr}_{L\left(\Lambda_{(2)} ; 13\right)} q^{L_{0}}\right)+q \widehat{\beta}_{12,-2}(\tau)+\frac{\left(q^{4} ; q^{4}\right)_{\infty}^{3}}{\left(q^{2} ; q^{2}\right)_{\infty}^{2}} \\
& \rho(q)=-\frac{1}{2} \cdot \widehat{\Theta}_{6}^{-1}\left(\operatorname{tr}_{L\left(\Lambda_{(-1)} ; 7\right)} q^{L_{0}}+\operatorname{tr}_{L\left(\Lambda_{(1)} ; 7\right)} q^{L_{0}}\right)+\frac{1}{2} \widehat{\beta}_{6,-1}(\tau) \\
& +\quad \frac{3}{2} \frac{\left(q^{6} ; q^{6}\right)_{\infty}^{4}}{\left(q^{2} ; q^{2}\right)_{\infty}\left(q^{3} ; q^{3}\right)_{\infty}^{2}} \\
& \xi(q)=1+2 q^{2} \cdot \widehat{\Theta}_{18}^{-1} \cdot \operatorname{tr}_{L\left(\Lambda_{(3)} ; 19\right)} q^{L_{0}}-2 q \widehat{\psi}_{18,3}(\tau) \\
& \sigma(-q)=q^{2} \cdot \widehat{\Theta}_{36}^{-1}\left(q^{\frac{3}{2}} \cdot \operatorname{tr}_{L\left(\Lambda_{(3)} ; 37\right)} q^{L_{0}}+q^{-\frac{3}{2}} \cdot \operatorname{tr}_{L\left(\Lambda_{(15)} ; 37\right)} q^{L_{0}}\right) \\
& -q^{2} \widehat{\beta}_{36,3}(\tau)+\frac{\left(q^{2} ; q^{2}\right)_{\infty}^{3}\left(q^{12} ; q^{12}\right)_{\infty}^{3}}{(q ; q)_{\infty}\left(q^{4} ; q^{4}\right)_{\infty}^{2}\left(q^{6} ; q^{6}\right)_{\infty}^{2}}
\end{aligned}
$$


TABLE 2. Character formulas for order 5 mock $\theta$-functions

\begin{tabular}{|c|c|c|}
\hline$f_{0}(q)$ & $=$ & $\begin{array}{l}-2 q^{2} \cdot \widehat{\Theta}_{30}^{-1}\left(q^{\frac{3}{2}} \cdot \operatorname{tr}_{L\left(\Lambda_{(4)} ; 31\right)} q^{L_{0}}+q^{-\frac{3}{2}} \cdot \operatorname{tr}_{L\left(\Lambda_{(14)} ; 31\right)} q^{L_{0}}\right) \\
2 q^{2} \widehat{\beta}_{30,4}+\theta_{4}\left(0, q^{5}\right) G(q)\end{array}$ \\
\hline$F_{0}(q)$ & $=$ & $\begin{array}{l}1+q \cdot \widehat{\Theta}_{15}^{-1}\left(q^{\frac{3}{4}} \cdot \operatorname{tr}_{L\left(\Lambda_{(2)} ; 16\right)} q^{L_{0}}+q^{-\frac{3}{4}} \cdot \operatorname{tr}_{L\left(\Lambda_{(7)} ; 16\right)} q^{L_{0}}\right) \\
q \widehat{\beta}_{15,2}-q \psi\left(q^{5}\right) H\left(q^{2}\right)\end{array}$ \\
\hline$\phi_{0}(-q)$ & $\begin{aligned}= & \\
& +\end{aligned}$ & $\begin{array}{l}-q \cdot \widehat{\Theta}_{15}^{-1}\left(q^{\frac{3}{4}} \cdot \operatorname{tr}_{L\left(\Lambda_{(2)} ; 16\right)} q^{L_{0}}+q^{-\frac{3}{4}} \cdot \operatorname{tr}_{L\left(\Lambda_{(7)} ; 16\right)} q^{L_{0}}\right) \\
q \widehat{\beta}_{15,2}+j\left(-q^{2}, q^{5}\right) G\left(q^{2}\right)\end{array}$ \\
\hline$\psi_{0}(q)$ & $=$ & $\begin{array}{l}q^{2} \cdot \widehat{\Theta}_{30}^{-1}\left(q^{\frac{3}{2}} \cdot \operatorname{tr}_{L\left(\Lambda_{(4)} ; 31\right)} q^{L_{0}}+q^{-\frac{3}{2}} \cdot \operatorname{tr}_{L\left(\Lambda_{(14)} ; 31\right)} q^{L_{0}}\right) \\
q^{2} \widehat{\beta}_{30,4}+q j\left(q, q^{10}\right) H(q)\end{array}$ \\
\hline$\chi_{0}(q)$ & $=$ & $\begin{array}{l}2+3 q \cdot \widehat{\Theta}_{15}^{-1}\left(q^{\frac{3}{4}} \cdot \operatorname{tr}_{L\left(\Lambda_{(2)} ; 16\right)} q^{L_{0}}+q^{-\frac{3}{4}} \cdot \operatorname{tr}_{L\left(\Lambda_{(7)} ; 16\right)} q^{L_{0}}\right) \\
\widehat{\beta}_{15,2}-j\left(q^{2}, q^{5}\right) G^{2}(q)\end{array}$ \\
\hline$f_{1}(q)$ & $=$ & $\begin{array}{l}-2 q^{3} \cdot \widehat{\Theta}_{30}^{-1}\left(q^{\frac{1}{2}} \cdot \operatorname{tr}_{L\left(\Lambda_{(-2)} ; 31\right)} q^{L_{0}}+q^{-\frac{1}{2}} \cdot \operatorname{tr}_{L\left(\Lambda_{(8)} ; 31\right)} q^{L_{0}}\right) \\
2 q^{3} \widehat{\beta}_{30,-2}+\theta_{4}\left(0, q^{5}\right) H(q)\end{array}$ \\
\hline$F_{1}(q)$ & $=$ & $\begin{array}{l}q \cdot \widehat{\Theta}_{15}^{-1}\left(q^{\frac{1}{4}} \cdot \operatorname{tr}_{L\left(\Lambda_{(-1)} ; 16\right)} q^{L_{0}}+q^{-\frac{1}{4}} \cdot \operatorname{tr}_{L\left(\Lambda_{(4)} ; 16\right)} q^{L_{0}}\right) \\
q \widehat{\beta}_{15,-1}+\psi\left(q^{5}\right) G\left(q^{2}\right)\end{array}$ \\
\hline$\phi_{1}(-q)$ & $=$ & $\begin{array}{l}q^{2} \cdot \widehat{\Theta}_{15}^{-1}\left(q^{\frac{1}{4}} \cdot \operatorname{tr}_{L\left(\Lambda_{(-1)} ; 16\right)} q^{L_{0}}+q^{-\frac{1}{4}} \cdot \operatorname{tr}_{L\left(\Lambda_{(4)} ; 16\right)} q^{L_{0}}\right) \\
-q^{2} \widehat{\beta}_{15,-1}-q j\left(-q, q^{5}\right) H\left(q^{2}\right)\end{array}$ \\
\hline$\psi_{1}(q)$ & $=$ & $\begin{array}{l}q^{3} \cdot \widehat{\Theta}_{30}^{-1}\left(q^{\frac{1}{2}} \cdot \operatorname{tr}_{L\left(\Lambda_{(-2)} ; 31\right)} q^{L_{0}}+q^{-\frac{1}{2}} \cdot \operatorname{tr}_{L\left(\Lambda_{(8)} ; 31\right)} q^{L_{0}}\right) \\
q^{3} \widehat{\beta}_{30,-2}+j\left(q^{3}, q^{10}\right) G(q)\end{array}$ \\
\hline$\chi_{1}(q)$ & $=$ & $\begin{array}{l}3 q \cdot \widehat{\Theta}_{15}^{-1}\left(q^{\frac{1}{4}} \cdot \operatorname{tr}_{L\left(\Lambda_{(-1)} ; 16\right)} q^{L_{0}}+q^{-\frac{1}{4}} \cdot \operatorname{tr}_{L\left(\Lambda_{(4)} ; 16\right)} q^{L_{0}}\right) \\
3 q \widehat{\beta}_{15,-1}+j\left(q, q^{5}\right) H^{2}(q)\end{array}$ \\
\hline
\end{tabular}


TABLE 3. Character formulas for order 7 mock $\theta$-functions

$\begin{aligned} \mathcal{F}_{0}(q)= & 2+2 q \cdot \widehat{\Theta}_{21}^{-1}\left(q^{\frac{5}{4}} \cdot \operatorname{tr}_{L\left(\Lambda_{(4)} ; 22\right)} q^{L_{0}}+q^{-\frac{5}{4}} \cdot \operatorname{tr}_{L\left(\Lambda_{(1)} ; 22\right)} q^{L_{0}}\right) \\ & -2 q \widehat{\beta}_{21,4}(\tau)-j^{2}\left(q^{3}, q^{7}\right)(q ; q)_{\infty}^{-1} \\ \mathcal{F}_{1}(q)= & 2 q^{2} \cdot \widehat{\Theta}_{21}^{-1}\left(q^{\frac{3}{4}} \cdot \operatorname{tr}_{L\left(\Lambda_{(1)} ; 22\right)} q^{L_{0}}+q^{-\frac{3}{4}} \cdot \operatorname{tr}_{L\left(\Lambda_{(8)} ; 22\right)} q^{L_{0}}\right) \\ & -2 q^{2} \widehat{\beta}_{21,1}(\tau)+q j^{2}\left(q, q^{7}\right)(q ; q)_{\infty}^{-1} \\ & \\ \mathcal{F}_{2}(q)= & 2 q^{2} \cdot \widehat{\Theta}_{21}^{-1}\left(q^{\frac{1}{4}} \cdot \operatorname{tr}_{L\left(\Lambda_{(-2)} ; 22\right)} q^{L_{0}}+q^{-\frac{1}{4}} \cdot \operatorname{tr}_{L\left(\Lambda_{(5)} ; 22\right)} q^{L_{0}}\right) \\ & -2 q^{2} \widehat{\beta}_{21,-2}(\tau)+j^{2}\left(q^{2}, q^{7}\right)(q ; q)_{\infty}^{-1}\end{aligned}$

TABLE 4. Character formulas for order 2 mock $\theta$-functions

$$
\begin{aligned}
& A\left(q^{2}\right)=q \cdot \widehat{\Theta}_{8}^{-1} \operatorname{tr}_{L\left(\Lambda_{(2)} ; 9\right)} q^{L_{0}}-q \widehat{\eta}_{8,2}(\tau)-q\left(-q^{2} ; q^{2}\right)_{\infty}\left(-q^{4} ; q^{4}\right)_{\infty}^{2}\left(q^{8} ; q^{8}\right)_{\infty} \\
& B(q)=\widehat{\Theta}_{4}^{-1} \operatorname{tr}_{L\left(\Lambda_{(0)} ; 5\right)} q^{L_{0}}-\widehat{\eta}_{4,0}(\tau) \\
& \mu\left(q^{4}\right)=-2 q \cdot \widehat{\Theta}_{4}^{-1} \operatorname{tr}_{L\left(\Lambda_{(0)} ; 5\right)} q^{L_{0}}+2 q \widehat{\eta}_{4,0}(\tau)+\frac{\left(q^{2} ; q^{2}\right)_{\infty}\left(q^{4} ; q^{4}\right)_{\infty}^{3}\left(q^{8} ; q^{8}\right)_{\infty}}{(q ; q)_{\infty}^{2}\left(q^{16} ; q^{16}\right)^{2}} 12
\end{aligned}
$$

TABLE 5. Character formulas for order 6 mock $\theta$-functions

$$
\begin{aligned}
\phi\left(q^{4}\right)= & -2 q \cdot \widehat{\Theta}_{12}^{-1} \operatorname{tr}_{L\left(\Lambda_{(4)} ; 13\right)} q^{L_{0}} \\
& +2 q \widehat{\eta}_{12,4}(\tau)+\frac{\left(q^{2} ; q^{2}\right)_{\infty}^{3}\left(q^{3} ; q^{3}\right)_{\infty}^{2}\left(q^{12} ; q^{12}\right)_{\infty}^{3}}{(q ; q)_{\infty}^{2}\left(q^{6} ; q^{6}\right)^{3}\left(q^{8} ; q^{8}\right)_{\infty}\left(q^{24} ; q^{24}\right)_{\infty}} \\
\psi\left(q^{4}\right)= & -q^{3} \cdot \widehat{\Theta}_{12}^{-1} \operatorname{tr}_{L\left(\Lambda_{(0)} ; 13\right)} q^{L_{0}}+q^{3} \widehat{\eta}_{12,0}(\tau) \\
& +q^{3} \frac{\left(q^{2} ; q^{2}\right)_{\infty}^{2}\left(q^{4} ; q^{4}\right)_{\infty}\left(q^{24} ; q^{24}\right)_{\infty}^{2}}{(q ; q)_{\infty}\left(q^{3} ; q^{3}\right)\left(q^{8} ; q^{8}\right)_{\infty}^{2}}
\end{aligned}
$$


TABLE 6. Character formulas for order 8 mock $\theta$-functions

$$
\begin{aligned}
& S_{0}\left(-q^{2}\right)=-2 q \cdot \widehat{\Theta}_{16}^{-1} \operatorname{tr}_{L\left(\Lambda_{(6)} ; 17\right)} q^{L_{0}}+2 q \widehat{\eta}_{16,6}(\tau)+\frac{j\left(-q, q^{2}\right) j\left(q^{6}, q^{16}\right)}{j\left(q^{2}, q^{8}\right)} \\
& S_{1}\left(-q^{2}\right)=-2 q \cdot \widehat{\Theta}_{16}^{-1} \operatorname{tr}_{L\left(\Lambda_{(2)} ; 17\right)} q^{L_{0}}+2 q \widehat{\eta}_{16,2}(\tau)+\frac{j\left(-q, q^{2}\right) j\left(q^{2}, q^{16}\right)}{j\left(q^{2}, q^{8}\right)}
\end{aligned}
$$

TABLE 7. Character formulas for order 10 mock $\theta$-functions

$$
\begin{aligned}
& \phi(q)=2 q \cdot \widehat{\Theta}_{10}^{-1} \operatorname{tr}_{L\left(\Lambda_{(1)} ; 11\right)} q^{L_{0}}-2 q \widehat{\eta}_{10,1}(\tau)+\frac{\left(q^{10} ; q^{10}\right)_{\infty}^{2} j\left(-q^{2}, q^{5}\right)}{\left(q^{5} ; q^{5}\right)_{\infty} j\left(q^{2}, q^{10}\right)} \\
& \psi(q)=2 q \cdot \widehat{\Theta}_{10}^{-1} \operatorname{tr}_{L\left(\Lambda_{(3)} ; 11\right)} q^{L_{0}}-2 q \widehat{\eta}_{10,3}(\tau)-q \frac{\left(q^{10} ; q^{10}\right)_{\infty}^{2} j\left(-q, q^{5}\right)}{\left(q^{5} ; q^{5}\right)_{\infty} j\left(q^{4}, q^{10}\right)} \\
& X\left(-q^{2}\right)=-2 q \cdot \widehat{\Theta}_{40}^{-1} \operatorname{tr}_{L\left(\Lambda_{(18)} ; 41\right)} q^{L_{0}}+2 q \cdot \widehat{\Theta}_{40}^{-1} \operatorname{tr}_{L\left(\Lambda_{(2)} ; 41\right)} q^{L_{0}} \\
& +2 q \widehat{\eta}_{40,18}(\tau)-2 q \widehat{\eta}_{40,2}(\tau) \\
& +\frac{\left(q^{4} ; q^{4}\right)_{\infty}^{2}\left(j\left(-q^{2}, q^{20}\right) j\left(q^{12}, q^{40}\right)+2 q\left(q^{40} ; q^{40}\right)^{3}\right)}{\left(q^{2} ; q^{2}\right)_{\infty}\left(q^{20} ; q^{20}\right)_{\infty}\left(q^{40} ; q^{40}\right)_{\infty} j\left(q^{8}, q^{40}\right)} \\
& \chi\left(-q^{2}\right)=-2 q^{3} \cdot \widehat{\Theta}_{40}^{-1} \operatorname{tr}_{L\left(\Lambda_{(14)} ; 41\right)} q^{L_{0}}-2 q^{5} \cdot \widehat{\Theta}_{40}^{-1} \operatorname{tr}_{L\left(\Lambda_{(6)} ; 41\right)} q^{L_{0}} \\
& +2 q^{3} \widehat{\eta}_{40,14}(\tau)+2 q^{5} \widehat{\eta}_{40,6}(\tau) \\
& +q^{2} \frac{\left(q^{4} ; q^{4}\right)_{\infty}^{2}\left(2 q\left(q^{40} ; q^{40}\right)_{\infty}^{3}-j\left(-q^{6}, q^{20}\right)^{2} j\left(q^{4}, q^{40}\right)\right)}{\left(q^{2} ; q^{2}\right)_{\infty}\left(q^{20} ; q^{20}\right)_{\infty}\left(q^{40} ; q^{40}\right)_{\infty} j\left(q^{16}, q^{40}\right)}
\end{aligned}
$$


TABLE 8. Auxiliary functions

$\begin{aligned} & G(q):=\prod_{n=1}^{\infty}\left(1-q^{5 n-4}\right)^{-1}\left(1-q^{5 n-1}\right)^{-1} \\ & H(q) \quad:=\prod_{n=1}^{\infty}\left(1-q^{5 n-2}\right)^{-1}\left(1-q^{5 n-3}\right)^{-1} \\ & \theta_{4}(0, q) \quad:=(q ; q)_{\infty}\left(q ; q^{2}\right)_{\infty} \\ & \psi(q) \quad:=\frac{\left(q^{2} ; q^{2}\right)_{\infty}}{\left(q ; q^{2}\right)_{\infty}} \\ & j(x, q) \quad:=(x ; q)_{\infty}\left(x^{-1} q ; q\right)_{\infty}(q ; q)_{\infty}\end{aligned}$

\section{REFERENCES}

[1] R.E. Borcherds, "Monstrous Moonshine and Monstrous Lie Superalgebras," Invent. Math. 109 (1992), 405-444. MR.1172696 (94f:11030)

[2] K. Bringmann and K. Ono, "The $f(q)$ mock theta function conjecture and partition ranks," Invent. Math. 165 (2006), 243-266. MR 2231957 (2007e:11127)

[3] K. Bringmann and K. Ono, "Some characters of Kac and Wakimoto and nonholomorphic modular functions," Math. Ann. 345 (2009), no. 3, 547-558. MR2534107 (2010f:11061)

[4] J.H. Conway and S.P. Norton, "Monstrous Moonshine," Bull. London Math. Soc. 11 (1979), 308-339. MR.554399 (81j:20028)

[5] B. Gordon and R. McIntosh, "A survey of mock-theta functions, I.," preprint, (2009).

[6] V. Kac, "Infinite-dimensional Lie algebras and Dedekind's eta function", Funct. Anal. Appl. 8 (1974), 68-70. MR0374210 (51:10410)

[7] V. Kac, Infinite-dimensional Lie algebras, Third edition. Cambridge University Press, Cambridge (1990). MR.1104219 (92k:17038)

[8] V. Kac and M. Wakimoto, "Modular invariant representations of infinite-dimensional Lie algebras and superalgebras," Proc. Nat. Acad. Sci. U.S.A. 85 (1988), no. 14, 4956-4960. MR949675 (89j:17019)

[9] V. Kac and M. Wakimoto, "Integrable highest weight modules over affine superalgebras and number theory," Lie theory and geometry, Progr. Math., 123, Birkhauser Boston, Boston, MA (1994), 415-456. MR.1327543 (96j:11056)

[10] V. Kac and M. Wakimoto, "Integrable highest weight modules over affine superalgebras and Appell's function," Comm. Math. Phys. 215 (2001), no. 3, 631-682. MR.1810948 (2001j:17017)

[11] S. Kang, "Mock Jacobi forms in basic hypergeometric series," Compositio Math. 145 (2009), no. 3, 553-565. MR.2507741 (2010f:33022)

[12] J. Lepowsky and S. Milne, "Lie algebraic approaches to classical partition identities," Advances in Mathematics 29 (1978), 15-59. MR501091 (82f:17005)

[13] J. Lepowksy and S. Milne, "Lie algebras and classical partition identities," Proc. Nat. Acad. Sci. U.S.A. 75 (1978), no. 2, 578-579. MR0491467 (58:10713)

[14] J. Lepowsky and R.L. Wilson, "The Rogers-Ramanujan identities: Lie theoretic interpretation and proof," Proc. Nat. Acad. Sci. U.S.A. 78 (1981), no. 2, part 1, 699-701. MR605423 (82a:17004)

[15] R. McIntosh, "Divisor sums and theta functions," in preparation. 
[16] K. Ono, "Unearthing the visions of a master: Harmonic Maass forms and number theory," Harvard-MIT Current Developments in Mathematics, 2008, 347-454, Int. Press, Somerville, MA, 2009. MR2555930

[17] A. Rocha-Caridi, "Vacuum vector representations of the Virasoro algebra," in Vertex operators in mathematics and physics (Berkeley, Calif., 1983), 451-473, Math. Sci. Res. Inst. Publ., 3, Springer, New York (1985). MR781391 (87b:17011)

[18] M. Wakimoto, "Representation theory of affine superalgebras at the critical level," Proc. of the ICM, Vol. II (Berlin, 1998), Doc. Math. (1998), Extra Vol. II, 605-614. MR1648109 (99k:17045)

[19] D. Zagier, "A proof of the Kac-Wakimoto affine denominator formula for the strange series," Math. Res. Lett. 7 (2000), no. 5-6, 597-604. MR.1809285 (2001m:11064)

[20] S.P. Zwegers, "Mock theta functions," Ph.D. Thesis, Universiteit Utrecht (2002).

Department of Mathematics, University of Wisconsin, Madison, Wisconsin 53706

E-mail address: folsom@math.wisc.edu

Current address: Department of Mathematics, Yale University, New Haven, Connecticut 06520

E-mail address: amanda.folsom@yale.edu 